$$
\begin{aligned}
& E\left\{\left(x_{1}-a_{1}\right)^{k}\left(x_{2}-a_{2}\right)^{l} \cdots\left(x_{r}-a_{r}\right)^{m}\right\} \\
& \left.\quad=\left(p_{1} e^{D_{1}}+p_{2} e^{D_{2}}+\cdots+p_{r} e^{D_{r}}\right)^{n} \cdot x_{1}^{k} x_{2}^{l} \cdots x_{r}^{m}\right]_{x_{r}=-a_{r}}^{x_{1}=-a_{1}},
\end{aligned}
$$

where $D_{1}=\partial / \partial x_{1}$.

WAShington, D. C.

\title{
ON A RESULTANT CONNECTED WITH FERMAT'S LAST THEOREM
}

\section{BY EMMA LEHMER}

E. Wendt* seems to have been the first to introduce the resultant of $x^{n}=1$ and $(x+1)^{n}=1$ in connection with Fermat's Last Theorem. This resultant can be expressed by means of the following circulant of binomial coefficients

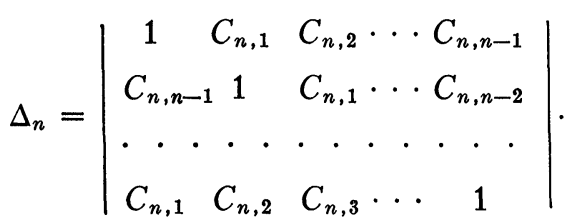

In his book on Fermat's Last Theorem Bachmann $\dagger$ proved that if $p$ is an odd prime and if $\Delta_{p-1}$ is not divisible by $p^{3}$, then Fermat's equation $x^{p}+y^{p}+z^{p}=0$ has no solution $(x, y, z)$ prime to $p$.

S. Lubelsky $\ddagger$ proved in a recent paper, using the distribution of quadratic residues, that if $p \geqq 7, \Delta_{p-1}$ is not only divisible by $p^{3}$, but by $p^{8}$, thus annulling Bachmann's criterion except for $p=3$ and $p=5$.

We shall now show how, by a straightforward manipulation with the above determinant, one can prove much more.

THEOREM $1 . \Delta_{p-1}$ is divisible by $p^{p-2} q_{2}$ for every prime $p$, where $q_{2}$ is the Fermat quotient $\left(2^{p-1}-1\right) / p$.

* Journal für Mathematik, vol. 113 (1894), pp. 335-347.

$\dagger$ Das Fermatproblem, 1919, p. 59.

$\ddagger$ Prace Matematyczno-Fizyczne, vol. 42 (1935), pp. 11-44. 
Proof. First add to each element of the last column of $\Delta_{p-1}$ the corresponding element of all the other columns. The elements of the last column now become equal to

$$
1+C_{p-1,1}+C_{p-1,2}+\cdots+C_{p-1, p-2}=2^{p-1}-1 .
$$

Next increase each element of the first $p-3$ columns by the element immediately to the right of it. All the elements of the first $p-3$ columns are now of the form

$$
C_{p-1, k}+C_{p-1, k+1}=C_{p, k+1}=p I_{k}, \quad(k=0,1, \cdots, p-2) .
$$

Since, as is well known, $I_{k}$ is an integer for $p$ a prime, it follows that $p$ is a factor of each of the first $p-3$ columns. Also $\left(2^{p-1}-1\right)$ comes out of the last column, and hence $\Delta_{p-1}$ is divisible by $\left(2^{p-1}-1\right) p^{p-3}=p^{p-2} q_{2}$, which is the theorem.

For example, if $p=5$,

$$
\begin{aligned}
\Delta_{4} & =\left|\begin{array}{rrrr}
1 & 4 & 6 & 4 \\
4 & 1 & 4 & 6 \\
6 & 4 & 1 & 4 \\
4 & 6 & 4 & 1
\end{array}\right|=\left|\begin{array}{llll}
1 & 4 & 6 & 15 \\
4 & 1 & 4 & 15 \\
6 & 4 & 1 & 15 \\
4 & 6 & 4 & 15
\end{array}\right| \\
& =\left|\begin{array}{rrrr}
5 & 10 & 6 & 15 \\
5 & 5 & 4 & 15 \\
10 & 5 & 1 & 15 \\
10 & 10 & 4 & 15
\end{array}\right|=15 \cdot 5^{2}\left|\begin{array}{cccc}
1 & 2 & 6 & 1 \\
1 & 1 & 4 & 1 \\
2 & 1 & 1 & 1 \\
2 & 2 & 4 & 1
\end{array}\right|=-5^{3} \cdot 3 .
\end{aligned}
$$

It is interesting to notice that although Theorem 1 is in no way dependent on the solvability of Fermat's equation, nevertheless it enables us to replace Bachmann's criterion by the following one.

If $\Delta_{p-1}$ is not divisible by $p^{p-1}$, then $x^{p}+y^{p}+z^{p}=0$ has no solution $(x, y, z)$ prime to $p$.

This in fact is merely a restatement of Wiefrich's criterion,* which states that if $p$ does not divide $q_{2}$, Fermat's equation has no solution in integers prime to $p$.

For example, since we have seen that $\Delta_{4}$ is not divisible by $5^{4}$, it follows from our criterion that $x^{5}+y^{5}+z^{5}=0$ has no solutions $(x, y, z)$ prime to 5 .

* Journal für Mathematik, vol. 136 (1909), pp. 293-302. 
Before proceeding further it may be of interest to give a short, though perhaps less elementary proof of Theorem 1, based directly on the definition of the resultant of two polynomials as the product $f\left(\alpha_{1}\right) \cdot f\left(\alpha_{2}\right) \cdots f\left(\alpha_{n}\right)$, where $f$ is one polynomial and the $\alpha$ 's are roots of the other.

$$
\Delta_{p-1}=\prod_{j=1}^{p-1}\left[\left(\epsilon_{j}+1\right)^{p-1}-1\right],
$$

where $\epsilon_{j}$ are all the $(p-1)$ st roots of unity.

For $\epsilon_{j}=1$, we get the factor $2^{p-1}-1$, and for $\epsilon_{j}=-1$, we get -1 . For $\epsilon_{j}=\epsilon$, any complex root of unity, we have

$$
(\epsilon+1)^{p-1}-1=C_{p-1,1} \epsilon+C_{p-1,2} \epsilon^{2}+\cdots+\epsilon^{p-1} .
$$

But since, for $p$ a prime,

$$
C_{p-1, k}=(-1)^{k}+p c_{k},
$$

where the $c$ 's are integers, ${ }^{*}$ we have

$$
\begin{aligned}
(\epsilon+1)^{p-1}-1 & =-\epsilon+\epsilon^{2}-\epsilon^{3}+\cdots+\epsilon^{p-1}+p f(\epsilon) \\
& =\epsilon\left(\epsilon^{p-1}-1\right) /(\epsilon+1)+p f(\epsilon)=p f(\epsilon),
\end{aligned}
$$

where $f(x)$ is a polynomial with integral coefficients. Hence

$$
\Delta_{p-1}=-\left(2^{p-1}-1\right) \prod_{\epsilon_{j} \neq \pm 1} p f\left(\epsilon_{j}\right)=-\left(2^{p-1}-1\right) \cdot p^{p-3} \prod f\left(\epsilon_{j}\right),
$$

where $\prod f\left(\epsilon_{j}\right)$ is an integral symmetric function of the roots of $\left(x^{p-1}-1\right) /\left(x^{2}-1\right)$ and hence an integer. Hence $\Delta_{p-1}$ is divisible by $p^{p-2} q_{2}$.

In comparing Theorem 1 with that of Lubelsky we see that Theorem 1 says more, except for $p=7$, in which case Theorem 1 guarantees divisibility of $\Delta_{6}$ by $7^{5}$ instead of $7^{8}$. On examining this case more closely we find that as a matter of fact $\Delta_{6}=0$. Indeed, we have in general the following theorem.

THEOREM 2. $\Delta_{n}=0$ if and only if $n=6 k$.

PRoof. In order that $\Delta_{n}=0$ it is both necessary and sufficient that $x^{n}=1$ and $(x+1)^{n}=1$ have a root $\rho$ in common. But the roots of $x^{n}=1$ are the $n$th roots of unity. Hence we can write

* Lucas, American Journal of Mathematics, vol. 1 (1878), pp. 229-230. 
$\rho=\cos \theta+i \sin \theta$, but since at the same time $\rho$ is a root of $(x+1)^{n}=1$, we have

$$
|\rho+1|=1=(\cos \theta+1)^{2}+\sin ^{2} \theta=2+2 \cos \theta,
$$

or $\cos \theta=-1 / 2$ and $\theta= \pm 2 \pi / 3$. This condition will be satisfied if and only if $\rho=\omega$ or $\omega^{2}$, while $(\rho+1)=-\omega^{2}$ or $-\omega$, and hence $(\rho+1)^{n}=1$ if and only if $n$ is a multiple of 6 .

One can easily show by adding to each element of the first and second column of $\Delta_{6 k}$ (written in determinant form) the corresponding elements of every third column, that the elements of the two resulting columns will be equal and hence that $\Delta_{6 k}=0$, but the writer has not been able to show from the circulant definition of $\Delta_{n}$ that $\Delta_{n} \neq 0$ if $n$ is not a multiple of 6 .

In conclusion we give a short table of $\Delta_{p-1}$.

$$
\begin{aligned}
& p \quad \Delta_{p-1} \\
& 3-3=-\left(2^{2}-1\right) \\
& 5-375=-\left(2^{4}-1\right) \cdot 5^{2} \\
& 7 \quad 0 \\
& 11-210736858987743=-\left(2^{10}-1\right) \cdot 11^{8} \cdot 31^{2} \\
& 130 \\
& 17-1562716 \quad 604038367719 \quad 196 \quad 682456 \quad 673 \quad 375= \\
& -\left(2^{16}-1\right) \cdot 17^{14} \cdot\left(3^{3} \cdot 5 \cdot 7^{3} \cdot 257\right)^{2}
\end{aligned}
$$

It appears from this table of $\Delta_{p-1}$, and can be shown without difficulty for any even $n$, that $\Delta_{n}$ is $-\left(2^{n}-1\right)$ times a perfect square. It can also be shown that $\Delta_{d}$ divides $\Delta_{n}$ if $d$ divides $n$.

Bethlehem, Pennsylvania 